\title{
THE STUDY OF DEACTIVATION AND REGENERATION OF A FLUID CRACKING ZEOLITE CATALYSTS
}

I. P. OKOYE

(Received 29, May 2008; Revision Accepted 15, August 2008)

\section{ABSTRACT}

The catalytic activities of modified and unmodified sodium Y-Zeolites catalysts was investigated. Modification by framework dealumination and gallium oxide incorporation led to an increase in catalytic activity. The catalytic activities of both parent and the modified samples were reduced in a time dependent manner due to coke deposition. Incorporation of gallium oxide altered the reaction pathways of $\mathrm{C}_{8}$ intermediates from n-butane reactions, thus producing more aromatic products. However, $\mathrm{Ga}_{2} \mathrm{O}_{3}$ in excess of $1 \%$ resulted in a large decrease of catalytic activity due to loss in Bronsted acidity. As Bronsted sites are removed, direct protonation of the alkane became difficult and catalytic activity was lowered at high $\mathrm{Ga}_{2} \mathrm{O}_{3}$ loading. The oxygen adsorption isotherms of the various coked catalysts showed that the deactivation process involved blockage of the zeolite pore sites by the deposited coke. Interestingly, regeneration of the coked sample was seen to completely restore the catalytic activity of both samples.

KEY WORDS: Zeolite catalyst, n-butane, regeneration, coke, adsorption, hydrocarbons, cracking

\section{INTRODUCTION}

In recent years molecular sieve catalysts have assumed an important role in industrial catalysis. The introduction of acid faujasite zeolites into industrial catalysts for fluid catalytic cracking in 1962 was one of the formost event and a historical landmark in zeolite catalysts (Magee and Blazek 1976). The applications of zeolite catalysts are expanding from the traditional petroleum refining to new improved fuel processing and to new roles in both petrochemical and chemical industries, (Venuto and Habib, 1979; Carson et. al 1988). The use of zeolites as catalysts is mainly due to the presence of Bronsted and Lewis acid sites inside the pore channels. These active Bronsted sites result from the hydroxyl groups associated with framework aluminum in the zeolite structures.

The conversion of simple hydrocarbons over zeolite catalysts are assumed to follow simple first order kinetics. However, early work on the kinetics of paraffin cracking on amorphous silica-alumina catalysts showed that simple first- order kinetic expression can only approximate experimental results. More exact theoretical expressions were formulated by assuming the competitive adsorption of reaction products on active sites, (Atkins and Pakins 2002). Results from nhexadecane cracking were successfully fitted by such expressions where the general Langmuir isotherm was used to represent the adsorption of all components present in the reaction mixture, (Corma et.al 1998; Caeiro et al 2007). One of the major problems associated with the use of zeolites as catalysts in hydrocarbon conversion is coke formation, which reduces the activity of the catalysts. Deactivation by poisoning and pore plugging has been attributed to coke formation/deposit during cracking reactions, (Gates et al 1979 and Haag 1994).

Deactivation modeling by poisoning and pore mouth blockage has received some theoretical treatment in the recent past,(Carbrol and Oberlin 1984; Caeiro et.al 2007). However, experimental approach is scanty and little information is available to explain the mechanism of catalyst deactivation due to coke build up or coke poisoning. Evidence from literature indicates that increased catalyst site density may favour increased rate of coke formation, (Gates et.al 1979). A scheme for the coke formation process has been reported by Asprey et.al 1999, and it was noted that coke formation over acidic solids comes mainly from the transformation of aromatic hydrocarbons- either initially present as reactant or generated from other precursors such as olefins via secondary reactions during the catalytic cracking processes. It was subsequently postulated that such adsorbed aromatics might form reactive ionic species which could couple with each other or with other aromatics or unsaturated molecules leading to a growing solid or semi-liquid polymer via dehydrogenation and condensation reactions, (Van Bokhoven et al 2001).

The aim of this work therefore, is to study experimentally the deactivation and coking processes of a modified and unmodified zeolite catalyst using a light paraffinic hydrocarbon. Sound knowledge of these processes would help in the effective regeneration and reuse of the zeolite cracking catalysts. The cracking of shorter chain hydrocarbons such as $\mathrm{n}-\mathrm{C}_{4}$ requires stronger acid site than the longer chain hydrocarbons. It is therefore expected that the cracking of $n-C_{4}$ over this class of zeolites would help to explain the rapid deactivation process inherent in zeolite and other related catalysts.

\section{EXPERIMENTAL}

\section{Sample Preparation}

The parent $\mathrm{Na}-\mathrm{Y}$ zeolite $(\mathrm{Si} / \mathrm{Al}=2.5)$ supplied by Cross field Industries Ltd UK, was first dealuminated using $\mathrm{SiCl}_{4}$. The dealumination achieved $\mathrm{Si} / \mathrm{Al}=7$ and designated as DY. The parent $\mathrm{Na}-\mathrm{Y}$ and DY samples 
were first converted into $\mathrm{NH}_{4}$-form by ion-exchange with $\mathrm{NH}_{4} \mathrm{NO}_{3}$ solution. About $5 \mathrm{~g}$ of DY sample was added to $100 \mathrm{ml}$ of gallium nitrate solution, containing a measured quantity of $\mathrm{Ga}\left(\mathrm{NO}_{3}\right)_{3} 6 \mathrm{H}_{2} \mathrm{O}$ calculated to give a desired amount of $1 \% \mathrm{Ga}_{2} \mathrm{O}_{3}$. The slurry was stirred at about $80^{\circ} \mathrm{C}$ and allowed to evaporate down to a thick paste, the point of incipient wetness. The sample was then dried at room temperature and finally dried overnight at $110^{\circ} \mathrm{C}$.

\section{Catalytic Cracking}

n-Butane cracking was carried out in a continuous flow (fixed bed) reactor. The reactor consists of a vertical quartz tube of $15 \mathrm{~mm}$ internal diameter fitted with a porous frit to support the catalyst pellates and also with a thermocouple well for measuring the bed temperature. About $100 \mathrm{mg}$ of each catalyst sample was loaded into the reactor and calcined in-situ, in a flow of nitrogen gas overnight at a temperature of $450^{\circ} \mathrm{C}$. This treatment converts the $\mathrm{NH}_{4}$ - form of the zeolite into its $\mathrm{H}$ - form. Testing of the catalyst was done by conversion of $n$-Butane at atmospheric pressure. The n-butane feed was supplied from a gas cylinder without any carrier gas. The reaction temperature was varied between 400 to $550^{\circ} \mathrm{C}$ where each sample was used at one particular temperature for about 3 hours. Changing the amount of catalyst or flow rate of the feed changed the contact time. The reaction products were analysed using Varian Vista 600 Gas Chromatography fitted with a thermal conductivity and flame ionization detectors. All the $\mathrm{GC}$ results were quantified using a Trivector Trilab 2000 multi-channel chromatography data station. The closed system allowed a mass balance to be made. However, only those experiments for which the mass balance were $100 \pm 10 \%$ were considered in this report.

\section{RESULTS AND DISCUSSION}

At the three temperatures studied for conversion of $n$-butane over $H / D Y$, the plot of $\ln (1 / 1-x)$ versus $W / F$ were linear (see figure1), such that the experimental data agrees well with the assumed pseudo-first order kinetic model. The first order rate constant, $k_{o}$ for the $n$ butane reaction can be calculated from the slope of the plot as shown in the equation below:

$\operatorname{Ln}(1 / 1-x)=k_{\circ}$ W $/ F_{\circ}$ where

$\mathrm{W}=$ weight of the catalyst in gram, $\mathrm{F}_{\mathrm{o}}=$ flow rate of $\mathrm{n}$ butane in mole/hour

$\mathrm{x}=$ fractional conversion of $\mathrm{n}$-butane and $\mathrm{k}_{\mathrm{o}}=$ rate constant

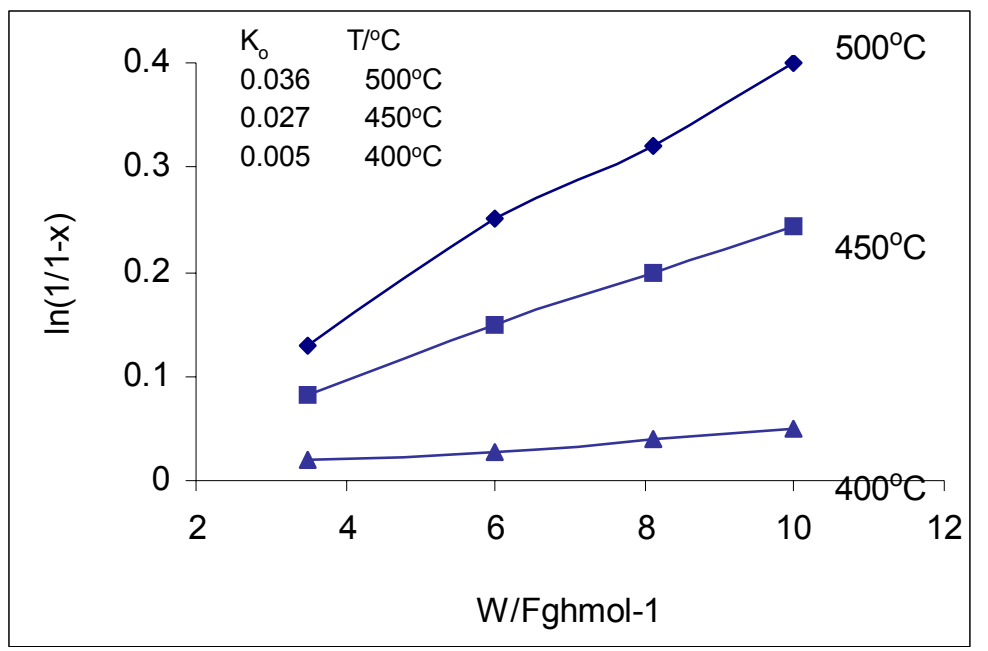

Figure 1: First order rate constant plot for n-butane conversion over $\mathrm{HD} / \mathrm{Y}$ zeolite

The rate constants obtained from the slopes are seen to increase with temperature in accordance with the Arrhenius law. The values of the apparent activation energies for both parent and modified zeolite samples are quite close $\left(120 \pm 6 \mathrm{kJmol}^{-1}\right)$ which is evidently an indication of a similar reaction mechanism. However the mechanism of paraffin cracking remains, to date, a controversial subject, Van Bokhoven et. al (2001). The modification of Y-zeolite by dealumination or $\mathrm{Ga}$ impregnation increases its catalytic activities in the present work. The parent material with $\mathrm{Si} / \mathrm{Al}=2.51$ is virtually inactive when compared with the H/DY and the gallated sample. By incorporation of small amount of $\mathrm{Ga}_{2} \mathrm{O}_{3}$, the activity increases more than two fold. (see Table1).

Table 1: Decay Parameters of parent and modified Y-zeolite catalyst

\begin{tabular}{l|lllll}
\hline & $\mathrm{HY}$ & $\mathrm{HDY}$ & $\mathrm{Ga}_{2} \mathrm{O}_{3} / \mathrm{DY}$ & & \\
\hline $\mathrm{Si} / \mathrm{Al}$ & 2.5 & 7 & $1 \%$ & $2 \%$ & $5 \%$ \\
$\%$ coke & 3.0 & 3.2 & 0.3 & 5.1 & 8.4 \\
Paraffin/olefin & 3.5 & 6.8 & 10.8 & 8 & 4 \\
$\mathrm{~K}_{\text {md }}$ & 0.88 & 1.9 & 2.3 & 2.45 & 2.12 \\
\hline Conversion\% & 2.50 & 10.48 & 17.50 & 7.51 & 4.77 \\
\hline
\end{tabular}

Reaction conditions: $450^{\circ} \mathrm{C}$ and $\mathrm{W} / \mathrm{F}_{\mathrm{o}}=3.5 \mathrm{ghmol}^{-1}$.

$\mathrm{K}_{\mathrm{md}}=$ Decay constant (Okoye, 2006) 
In paraffin cracking, coke has been identified as a secondary product, whose formation is accelerated by the olefin formed during the first cracking step. From table 1 , the parent sample contains more coke but decays more slowly than the modified materials. This trend is easily explained by the fact that dealumination and gallation leads to a decrease in the number of acid sites responsible for coke formation. The result presented in table 1 show that the decay parameter $k_{m d}$ for the zeolite sample studied in this work, is inversely related to the paraffin-to-olefin ratio in the products, Okoye (2006). This indicates that the decay (deactivation) of these catalysts is invariably linked with the rate of olefin formation.

It is therefore clear that the amount of coke formed on the modified $Y$-zeolites, allowing for margin of error showed a direct relation between the amount of olefins and coke formed at a given conversion level of $n-$ butane. It is surprising to note that the gallated sample $\left(1 \% \mathrm{Ga}_{2} \mathrm{O}_{3} / \mathrm{DY}\right.$ ) with low $\mathrm{Ga}$ loading (table 1) contains less coke than the non-gallated sample. The amount of coke deposit increases with increase in gallium oxide contents possible because of higher hydrogen transfer activity. Analysis using TEM/EDAX techniques revealed that the gallium oxide is homogeneously dispersed within the zeolite cavity at low oxide content. TEM analysis showed no evidence of extensive sintering, however, there was evidence of surface enrichment of gallium oxide for $5 \%$ and $10 \% \quad \mathrm{Ga}_{2} \mathrm{O}_{3}$ loading. This surface enrichment (poor dispersion) at higher gallium oxide loading $\left(5-10 \% \quad \mathrm{Ga}_{2} \mathrm{O}_{3}\right)$ may account for the increase in coke formation and subsequent decrease in catalytic activity or the rapid deactivation.

The activities of both the parent and modified catalysts are reduced with increasing time-on-stream, due to the deposition of coke. Coking is less severe in modified Y-zeolites but the amount of coke deposit increases with increase of gallium oxide content. The observed phenomenon cannot be explained by chemical process alone, but can more likely be explained by assuming that the deactivation process also involves pore plugging or site blockage by coke.

To investigate this additional decay process further, a series of oxygen gas adsorption experiment were carried on coked and uncoked (fresh) catalyst samples at low temperature. Figure 2 shows the oxygen adsorption isotherms of the fresh and the coked catalyst (after reaction with $n$-butane). The isotherm of the fresh sample $(H D / Y)$ is nearly rectilinear which is characteristic of adsorption on microporous and crystalline solids. On the other hand, the isotherms of coked samples showed an increase in the flat region at the beginning of the isotherm, indicatives of progressive increase of pore plugging. The differences arising from the shapes of the isotherms as shown in figure 2 and table 2, strongly imply that the catalyst pores are being filled up by coke since the pore volume decreases with increase in coke content.

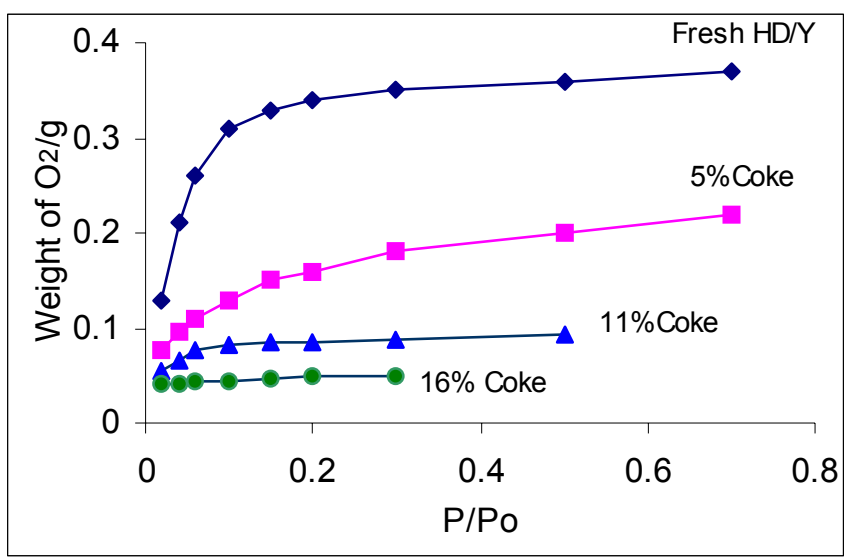

Figure 2: Oxygen adsorption isotherms for fresh and coked zeolite catalyst samples.

Table 2: The pore volumes of fresh and coked samples determined from the oxygen isotherms

\begin{tabular}{ll}
\hline Catalyst & Pore volume $/ \mathrm{cm}^{3} \mathrm{~g}^{-1}$ \\
\hline Fresh HD/Y & 0.26 \\
$5 \%$ Coke & 0.13 \\
$11 \%$ Coke & 0.10 \\
$16 \%$ Coke & 0.08 \\
\hline
\end{tabular}

\section{Regeneration of Deactivated Catalyst}

The two coked catalyst samples, $1 \%$ Ga/DY and H/DY were subjected to an in-situ regeneration by calcining overnight at about 15 hours in a flow of oxygen gas. Figure 3 shows the conversion of n-butane over each of the catalysts before and after regeneration. Regeneration is seen here to completely restore the catalytic activity of both catalysts. However, the activities of both catalysts are reduced with increasing time-onstream, due to the deposition of coke. Coking is very rapid especially during the first few minutes of the reaction, and this pattern is common to all the zeolite catalysts tested in this reaction. 


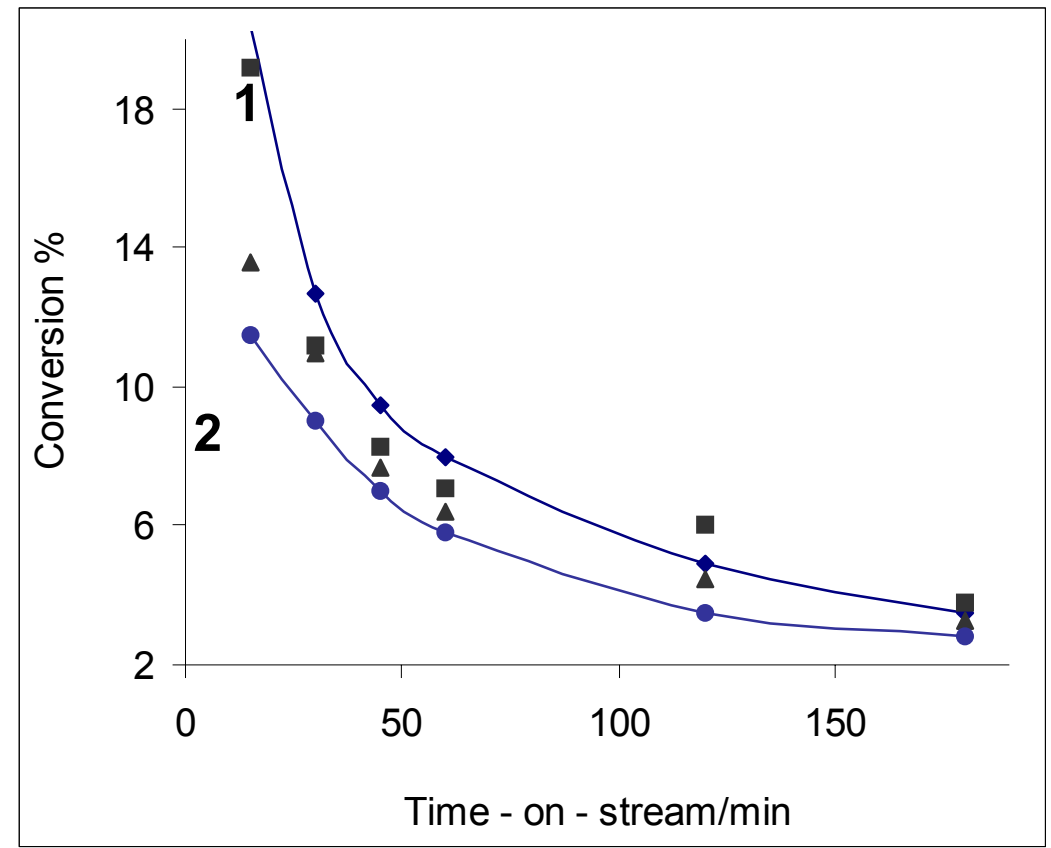

Figure 3: The plot of catalyst conversion versus time - on - stream, showing the effect of regeneration on catalytic activity at $500^{\circ} \mathrm{C}$ and $\mathrm{W} / \mathrm{F}=3.5 \mathrm{ghmol}^{-1}$

(1) $1 \% \mathrm{Ga} / \mathrm{DY} ; \bullet$ Fresh, - Regenerated (2) H/DY; $\Delta$ Fresh, • Regenerated

\section{ACKNOWLEDGEMENT}

Thanks to Chemistry Department, University of Manchester Institute of Science and Technology, UK for providing the laboratory facilities for the catalytic reactions.

\section{REFERENCES}

Asprey, S.P., Wojciechowski, B. W. and Peppley, B.A., 1999. "Kinetic Studies using Temp. Scanning and Steam reforming of Methanol". Appl. Catal. A. General, 179, 51-70.

Atkins, P. and Pakins, J. P., 2002. "Physical Chemistry" 7 th ed, NY, p1004-5.

Caeiro, G., Costa, A.F., Cerqueira, H.S, Mangnoux, P., Lopes, J.M., Martias , P. and Ramoa R. F., 2007. "Nitrogen poisoning effect on the catalytic cracking of gas oil". Appl. Catal. A, General 320, $8-15$.

Carbrol, R A and Oberlin, A., 1984. "Nature and Localization of poisoning carbonaceous matter in reforming catalyst". J. Catal. 89, $256-265$.

Carson, R., Cooke, E.M., Dwyer, J., Hinchliffe, A. and O'Malley, P. J., 1988. "Zeolites as catalysts, Sorbants and Detergent Builders," Extended Abstracts, page 151.

Corma, A., Monton, J. B., Martinez, V. and Orchilles, A. V., 1998. "Catalytic application of mesoporous molecular sieves to vapour-phase rearrangement of cyclohexane". Catal. Lett. 53(3):

Gates, B. S., Katzer, J. R. and Schult, G., 1979. "Chemistry of Catalytic Process." McGraw-Hill, Academic Press, NY.

Haag, W. O., 1994. In "Zeolite and related materials: State of the Art, Studies in Surface Science and Catalysis" (J.Weitkamp, H.J. Kalge and W. Holderich, eds.), 84, p.1375. Elsevier, Amsterdam, 1994.

Magee, J. S. and Blazek, J. J., 1976. Zeolite Chemistry and Catalysis, ACS Monogr. 171: 579 - 615.

Okoye, I P., 2006. Iterative estimation of kinetic and decay parameters of a fluid cracking catalyst. Scientia Africana 5 (2): 40 - 45.

Van Bokhoven, J.A., Tromp, M., Koiningsberger, D.C., Miller, J.T., Pieterse, J.A.Z., Lercher, J.A., Williams, and Kung, H. H., 2001. "An explanation for the enhanced activity for light alkane conversion in mildly steam dealuminated mordenite: the dominant role of adsorption". Journal of Catalysis, 202(1): $129-140$.

Venuto, P. B and Habib, E. T., 1979. "Fluid catalytic cracking with zeolite catalysts", Marcel dekker Inc. NY p156. 